ratio, serum protein and albumin concentration, and serum creatinine concentration. Diagnostic paracentesis was carried out and samples of ascitic fluid (AF) taken for cell count and differential, microscopy, culture and sensitivity, and AF protein and albumin concentration. CP and MELD scores were computed for all patients. MELD score was calculated using internet medcalculator. SBP was defined as the presence of $\mathrm{AF}$ neutrophil count of $\geq 250$ cells $/ \mathrm{mm}^{3}$. The concordance (c) statistics equivalent to the area under receiver operating characteristics curve was computed for CP and MELD scores respectively and compared using Delong's method.

Results Of the 170 patients, $64(37.6 \%)$ had SBP. One hundred and ten (64.7\%) had CP score B and 60 (35.3\%) had CP score of C. Mean CP score was $8.7 \pm 1.9$. Seventy five (44.1\%) had <10, 65 (38.2\%) had 10-19, 28 (16.5\%) had 20-29, $1(0.6 \%)$ had $30-39$ and 1 (0.6\%) had $\geq 40$ MELD scores respectively. Mean MELD score was $13.4 \pm 6.9$. The cstatistics for CP score and MELD score were 0.87 (95\%CI: $0.71-0.85)$ and $0.64(95 \% \mathrm{CI}: 0.56-0.73)$ respectively. The difference was statistically significant $(p=0.0004)$

Conclusions Child-Pugh score was superior to MELD score as a predictor of SBP in our study patients.

\section{IDDF2018-ABS-0068 A POTENTIAL LINK BETWEEN POLYCYSTIC OVARY SYNDROME AND NON-ALCOHOLIC FATTY LIVER DISEASE: AN UPDATE META- ANALYSIS}

${ }^{1}$ Xiaoyong Wang*, ${ }^{2}$ Jia Wu. 'Department of Gastroenterology, Changzhou No. 2 People's Hospital, Affiliated with Nanjing Medical University, China; 'Department of Gynecology, Changzhou No. 2 People's Hospital, Affiliated with Nanjing Medical University, China

\subsection{6/gutjin-2018-IDDFabstracts.200}

Background Polycystic ovary syndrome (PCOS) itself accounts for a high risk of developing non-alcoholic fatty liver disease (NAFLD). Alternatively, other specific factors in women with PCOS may contribute to this association, which presently remains unknown. Therefore, we aimed to shed some light on this issue, and thereby performed this meta-analysis.

Methods Relevant studies that were published before May 2017 were identified and retrieved from PubMed and Web of Science databases. Data were extracted, and the pooled odds ratios (ORs) and 95\% confidence intervals (95\% CIs) were calculated.

Results A total of 17 studies were included in this analysis. Compared to the control group, the risk of NAFLD in the PCOS group was higher $(\mathrm{OR}=2.25,95 \% \quad \mathrm{CI}=1.95-2.60)$. When stratified by BMI and geographic location, these results indicated that the frequency of NAFLD risk was significantly higher amongst obese subjects $(\mathrm{OR}=3.01,95 \% \mathrm{CI}=1.88-$ 4.82), non-obese subjects $(\mathrm{OR}=2.07,95 \% \quad \mathrm{CI}=1.12-3.85)$, subjects from Europe $(\mathrm{OR}=2.00,95 \% \mathrm{CI}=1.58-2.52)$, subjects from the Asia-Pacific Region, $(\mathrm{OR}=2.32,95 \% \mathrm{CI}=1.89-2.84)$ and subjects from America $(\mathrm{OR}=2.96,95 \% \mathrm{CI}=1.93-4.55)$, respectively. In addition, PCOS patients with hyperandrogenism (HA) had a significantly higher risk of NAFLD than controls $(\mathrm{OR}=3.31 ; 95 \% \mathrm{CI}=2.58-4.24)$. However, there was no association between PCOS patients without HA and a higher risk of NAFLD $(\mathrm{OR}=1.46 ; 95 \% \mathrm{CI}=0.55-3.87)$.

Conclusions The results of this meta-analysis suggest that PCOS is significantly associated with high risk of NAFLD. This association was independent of obesity and geographic region but might correlate with HA.

\section{IDDF2018-ABS-0078 LONG-TERM SURVIVAL AND PROGNOSTIC FACTORS OF HEPATOCELLULAR CARCINOMA AFTER RADIOFREQUENCY ABLATION WITH COOL-TIP ELECTRODE: PROSPECTIVE RESULT IN 105 PATIENTS}

${ }^{1}$ Nguyen Thi Thu Huyen*, ${ }^{2}$ Mai Hong Bang, ${ }^{2}$ Tran Van Riep, ${ }^{2}$ Nguyen Tien Thinh. ${ }^{1}$ Thai Nguyen University of Medicine and Pharmacy, Vietnam; ${ }^{2} 108$ Central Hospital, Vietnam

\subsection{6/gutjnl-2018-IDDFabstracts.201}

Background Radio-frequency ablation (RFA) has been indicated as a curative treatment for early-stage hepatocellular carcinoma (HCC). This study was to assess the long-term survival result and analyse risk factors of percutaneous Radiofrequency Ablation with Cool-tip in HCC patients.

Methods A prospective study involved 105 cirrhotic HCC patients (mean tumour size:32,5 $\pm 11,3 \mathrm{~mm}$ ) underwent percutaneous RFA using Cool-tip RF electrode (COOL-TIP E SERRIES,COVIDIEN) at the 108 hospital, from September 2012 to December 2017). The Kaplan-Meier curves and the multivariate Cox regression analysis were used to assess the prognostic factors.

Results The progression-free survival (PFS) was 23.6 \pm 1.2 months and the overall survival (OS) was $40.3 \pm 0.74$ months. The cumulative 1 year, 2 year, 3 year, 4 year survival probability were $98 \% ; 95.2 \% ; 87,3 \%$ and 70,1 respectively. The PFS was related to tumour morphology, HCC differentiation BCLC staging. The OS were affected by tumour characteristics, number of tumour, AFP response, and tumour complete response. Pre-treatment elevated AFP, multifocal HCCs, nonresponse AFP, in-complete tumour necrosis were negative prognostic factors for long-term survival. The OS and survival rates were also related to Child Pugh class and stage of tumour. At multivariate analysis (Cox Survival Analysis) tumour size and liver function (Chil-Pugh class) were independent significant predictors of overall patient survival.

Conclusions RFA with Cool-tip electrode is effective for HCC patients; the long-term survival result depends on some prognostic factors before treatment.

\section{IDDF2018-ABS-0079 THE SAFETY OF PERCUTANEOUS RADIOFREQUENCY ABLATION WITH COOL- TIP ELECTRODE FOR THE TREATMENT OF HEPATOCELLULAR CARCINOMA}

${ }^{1}$ Nguyen Thi Thu Huyen*, ${ }^{2}$ Mai Hong Bang, ${ }^{2}$ Tran Van Riep, ${ }^{2}$ Nguyen Tien Thinh. ${ }^{1}$ Thai Nguyen University of Medicine and Pharmacy, Vietnam; ${ }^{2} 108$ Central Hospital, Vietnam

\subsection{6/gutjnl-2018-IDDFabstracts.202}

Background Although ablation therapy has been accepted as a safe technique for treatment of unrespectable HCCs, investigation of its complications has been limited, and A physician who performs ablation treatment should be aware of the broad spectrum of complications. We aim to study the safety of percutaneous Radiofrequency Ablation with Cool-tip for the treatment of HCC.

Methods A prospective study involved 82 cirrhotic HCC patients (mean tumour size: $31,54 \pm 10,7 \mathrm{~mm}$ ) underwent percutaneous RFA using Cool-tip RF electrode (COOL-TIP E SERRIES, COVIDIEN) at the 108 hospital, from September 2012 to October 2016). Patients were evaluated during RFA treatment, throughout the immediate post RFA course, and 
then every 3 months after RFA to assess for the development of treatment-related complications.

Results A total 115 procedures were performed successfully. No death related to the technique. There were 3 cases with early complication (1 diaphragm perforation, 1 lesion abcess, 1 pleural effusion,) and tumour seeding developed in 2 patients. The rate of Post RFA syndrome was $67 \%$, but most of the symptoms were transient and self-limited.

Conclusions RFA using Cool-tip is a safe treatment for patients HCC.

\section{IDDF2018-ABS-0080 RISK FACTORS FOR LOCAL RECURRENCE IN THE TREATMENT OF RADIOFREQUENCY ABLATION WITH COOL-TIP ELECTRODE FOR HCC PATIENTS}

${ }^{1}$ Nguyen Thi Thu Huyen*, ${ }^{2}$ Mai Hong Bang, ${ }^{2}$ Tran Van Riep, ${ }^{2}$ Nguyen Tien Thinh. ${ }^{1}$ Thai Nguyen University of Medicine and Pharmacy, Vietnam; ${ }^{2} 108$ Central Hospital, Vietnam

\subsection{6/gutjnl-2018-IDDFabstracts.203}

Background Radiofrequency ablation (RFA) is one of the curative therapies for HCC patients. However, post-RFA local recurrence is a major factor limiting the outcome. The aim of this study was to evaluate the recurrent rate and analyse the risk factors for local recurrence of percutaneous Radiofrequency Ablation using Cool-tip electrode for the treatment of HCC.

Methods A prospective study involved 82 cirrhotic HCC patients (mean tumour size: 3,2 $\pm 1,1 \mathrm{~mm}$ ) underwent percutaneous RFA using Cool-tip RF electrode (COOL-TIP E SERRIES, COVIDIEN) at the 108 Hospital, from September 2012 to November 2017. We use single gauge, cluster, or multiple electrodes with an exposed needle tip of variable length $(2,3$ or $4 \mathrm{~cm}$ ). The rate of recurrence was recorded, and the prognostic factors for the tumour local recurrence were determined.

Results There were $37 / 75$ of patients presented recurrence after achieved complete response, including local recurrence in $11 / 75(14.7 \%)$ new nodule recurrence in 16/75 (21.3\%) and both local and new nodule recurrence in $7 / 75$ (9.3\%). The mean time of recurrence was 23 months (12-45 months). Tumour size $(3 \mathrm{~cm}-5 \mathrm{~cm})$, tumour location (close to vascular), size of ablative margin $(<0.5 \mathrm{~cm})$, high serum AFP level had a significant adverse prognostic factor for local tumour recurrence.

Conclusions Although RFA using Cool-tip is an effective treatment for local tumour control in HCC patients, the long term result depends on some prognostic factors before treatment.

\section{IDDF2018-ABS-0081 GRADUALLY DECREASING OF SERUM HBCRAG IN REAL-WORLD CHRONIC HEPATITIS B PATIENTS RECEIVING LONG- TERM NUCLEOT(S)IDE ANALOGUES-BASED THERAPY}

Menglan Wang*, Enqiang Chen, Chuanming Tao, Juan Liao, Taoyou Zhou, Juan Wang, Libo Yan, Dongbo Wu, Lingyao Du, Hong Tang. West China Hospital of Sichuan University, China

10.1136/gutjnl-2018-IDDFabstracts.204
Background This study aimed to investigate the detailed dynamics of $\mathrm{qHBcrAg}$ and influence factors of long-term qHBcrAg in a cohort of patients who had received over 8 years of continuous NAs therapy.

Methods This was a retrospective study. All patients were recruited from our previous published study, who started therapy with NAs between 2007 and 2008. Serum HBsAg and HBcrAg levels were quantitatively measured at baseline, the sixth month and each year of follow-up, using the stored serum samples.

Results Among the 94 patients, serum qHBcrAg presented a gradually decreasing trend from baseline to year 8 , either in $\mathrm{HBeAg}$-negative or $\mathrm{HBeAg}$-positive patients. Good correlation of $\mathrm{qHBcrAg}$ and $\mathrm{qHBsAg}$ was observed at baseline, but this correlation weakened remarkably during treatment. Serum qHBcrAg.

Conclusions Serum HBcrAg levels are varied during the course of antiviral therapy, and $\mathrm{qHBcrAg}$ may serve as a new important tool for the management of CHB.

\section{IDDF2018-ABS-0083 NONALCOHOLIC FATTY LIVER DISEASE IS ASSOCIATED WITH INCREASED ATRIAL FIBRILLATION RISK IN AN ELDERLY CHINESE POPULATION: A CROSS- SECTIONAL STUDY}

${ }^{1}$ Yu Zhang $*$, ${ }^{2}$ Peifei Li, ${ }^{2} Y_{\text {Li Liu, }}{ }^{2}$ Yue Pan, ${ }^{1}$ Lu Xu, ${ }^{3}$ Min Miao, ${ }^{4}$ Zhongwei Zhu, ${ }^{5} \mathrm{Chengfu} \mathrm{Xu}$ ${ }^{2}$ Lei Xu. ${ }^{1}$ Ningbo University, College of Medicine, Ningbo, China; ${ }^{2}$ Department of Gastroenterology, Ningbo First Hospital, Ningbo, China; ${ }^{3}$ Department of Gastroenterology, The Affiliated Hospital of Ningbo University, School of Medicine, Ningbo, China; ${ }^{4}$ Department of Gastroenterology, Zhenhai Lianhua Hospital, Ningbo, China; ${ }^{5}$ Department of Gastroenterology, The First Affiliated Hospital, College of Medicine, Zhejiang University, Hangzhou, China

\subsection{6/gutjnl-2018-IDDFabstracts.205}

Background Atrial fibrillation and nonalcoholic fatty liver disease are two pathological conditions that are highly prevalent worldwide and share multiple CVD risk factors. There is rare research performed among elderly adults.

Aims We conducted this cross-sectional analysis of elderly adults ( $\geq 65$ years) to investigate the association between atrial fibrillation and nonalcoholic fatty liver disease.

Subjects We conducted a cross-sectional study of the elderly adults ( $\geq 65$ years old) who had undergone an annual physical examination at Zhenhai Lianhua Hospital, Ningbo, China in 2014. 1688 participants (930 males and 758 females) with a median age of 72 (68-76) years were included in this analysis. This study excluded the following participants: (1) those with unknown alcohol intake or excessive alcohol intake; (2) those with unknown BMI or BMI2; (3) those with incomplete basic physical data; (4) those with missing liver ultrasonic diagnosis; (5) those with unknown causes of chronic liver disease. This study was approved by the Hospital Ethics Committee. All the participants were verbally informed and agreed to participate in the study. Written informed consent was not required for the observational nature of the study. Methods We analysed clinical data of the elderly adults $(\geq 65$ years) who took health examination in Zhenhai Lianhua hospital, Ningbo, China in 2014.

Results 522 of the 1688 participants were diagnosed with nonalcoholic fatty liver disease, and 39 participants were confirmed as having atrial fibrillation. Nonalcoholic fatty liver disease was associated with risk factors for AF in the elderly Chinese population (OR 1.95, 95\% CI 1.03-3.69). 